\title{
Chaos modified wall formula damping of the surface motion of a cavity undergoing fissionlike shape evolutions
}

\author{
Santanu Pal* and Tapan Mukhopadhyay ${ }^{\dagger}$ \\ Variable Energy Cyclotron Centre, 1/AF Bidhan Nagar, Calcutta 700 064, India
}

(May 13, 2018)

\begin{abstract}
The chaos weighted wall formula developed earlier for systems with partially chaotic single particle motion is applied to large amplitude collective motions similar to those in nuclear fission. Considering an ideal gas in a cavity undergoing fissionlike shape evolutions, the irreversible energy transfer to the gas is dynamically calculated and compared with the prediction of the chaos weighted wall formula. We conclude that the chaos weighted wall formula provides a fairly accurate description of one body dissipation in dynamical systems similar to fissioning nuclei. We also find a qualitative similarity between the phenomenological friction in nuclear fission and the chaos weighted wall formula. This provides further evidence for one body nature of the dissipative force acting in a fissioning nucleus.
\end{abstract}

PACS numbers:24.60.Lz,21.60.Ev,05.45.+b,25.70.Lm

*Electronic address:santanu@veccal.ernet.in

${ }^{\dagger}$ Electronic address:tkm@veccal.ernet.in 


\section{INTRODUCTION}

It is now well established [1 9] that a dissipative force operates in the dynamics of a fissioning nucleus as it descends from the saddle point to the scission. Since the fission process is expected to follow the mean field dynamics when the excitation energy of the nucleus is not too high, the physical processes that give rise to dissipation in fission are usually identified as one body mechanisms. The systematics of experimental mean kinetic energies of the fission fragments were fairly well reproduced [1,2]9 using an one body dissipative force in classical dynamical calculations. However it has also been observed that the mean kinetic energy of the fission fragments is not very sensitive to the details of the dissipative forces. Though two body processes are less favoured compared to one body processes in the long mean free path dominated mean field regime, both one and two body dissipations in classical dynamical calculations have been found 2,91 to describe the systematics of experimental mean kinetic energies. Nix and Sierk, on the other hand, suggested 3 3 . in their analyses of mean fragment kinetic energy data that the effective dissipation is about four times weaker than that predicted by the wall plus window formula of one body dissipation.

It has been pointed out [6 8 ] in recent years that prescission neutron and $\gamma$-multiplicities are sensitive to the details of the friction force in nuclear fission. In particular, it was shown [8] that one body dissipation is preferred to two body viscosity in order to describe the neutron multiplicity data. It was further observed [6] that a shape dependent dissipative force is essential for simultaneous reproduction of experimental values of prescission $\gamma$-multiplicities and fission probabilities. Specifically, it has been observed [6,7] that the dissipative force in a compact configuration of the fissioning compound nucleus should be much smaller than that predicted by the wall formula of one body dissipation.

The wall formula was developed by Blocki et al. [1] in a simple classical picture of one body dissipation in which energy is transferred from the nuclear surface motion to the nucleon motion as a result of frequent collisions of the nucleons with the nuclear surface. According to the wall formula, the rate of energy dissipation is given as

$$
\dot{E}_{\mathrm{WF}}(t)=\rho \bar{v} \int \dot{n}^{2} d \sigma,
$$

where $\dot{n}$ is the normal component of the surface velocity at the surface element $d \sigma$ while the nuclear mass density and the average nucleon speed inside the nucleus are denoted by $\rho$ and $\bar{v}$ respectively. The wall formula was also obtained from a formal theory of one body dissipation based on classical linear response techniques [10].

The wall formula was originally derived for idealized systems employing a number of simplifying assumptions such as approximating the nuclear surface by a rigid wall and considering only adiabatic collective motions. The validity of these assumptions were scrutinised [11,12] in the framework of random phase approximation (RPA) damping. It was shown 11.,12 that in the limiting situation where the above assumptions are realized, RPA damping coincides with the wall formula. These works further brought out the importance of including the $2 \mathrm{p} 2 \mathrm{~h}$ states for realistic applications.

We are however of the opinion that since the wall formula captures all the essential ingredients of dissipation under mean field dynamics, it is still possible to improve upon the dissipation rate given by Eq.(1.1) by examining its various assumptions. One such assumption of the wall formula concerns the randomization of the particle motion. It is assumed [1] that successive collisions of a nucleon with the one body potential give rise to a velocity distribution which is completely random. In other words, a complete mixing in the classical phase space of the particle motion is required. We have recently discussed [13] the implications of these assumptions and obtained a modification of the wall formula by relaxing the full randomization assumption in order to make it applicable to systems in which the mixing in phase space is partial. Considering chaotic particle trajectories which arise due to irregularity in the shape of the one body potential and which are responsible for irreversible energy transfer, we modified the wall formula in Ref. [13] as,

$$
\dot{E}_{\mathrm{CWWF}}=\mu \rho \bar{v} \int \dot{n}^{2} d \sigma,
$$

where $\mu$ is a measure of chaos (chaoticity) in the single particle motion. The chaoticity $\mu$ is 1 for cavities of highly irregular shapes in which most of the particles move in a chaotic fashion, and one recovers the original wall formula given by Eq.(1.1) for such systems. Eq.(1.2) becomes useful however for systems in which the particle motion is partially chaotic giving rise to a chaoticity less than 1. In fact, Eq.(1.2) gave a quite successful description [14] of energy transferred to a gas from the of its container oscillating with small amplitudes. In what follows, we shall use the term "chaos weighted wall formula" (CWWF) for Eq.(1.2) in order to distinguish it from the original wall formula (WF) given by Eq.(1.1).

In the present work, we shall consider an ideal gas in a cavity which will be subjected to a time dependent quadrupole deformation. We shall calculate the energy transferred to the particles from the wall motion. We shall then compare 
this transferred energy with that predicted by the chaos weighted wall formula (Eq.(1.2)). In this model, the cavity will be progressivly deformed until the neck radius vanishes. The motion will be thus tailored to resemble that of a fissioning nucleus as it descends from saddle to scission. Our motivation in this work is twofold. First, we wish to test the applicability of the chaos weighted wall formula for large amplitude motions such as fission. Second, we would like to compare the chaos weighted wall formula with the phenomenological reduced friction suggested by Fröbrich et al. [7]. We shall find that the chaos weighted wall formula can account for the energy damping fairly accurately in large amplitude collective motions in an ideal gas. We shall also observe a qualiatative similarity between the chaos weighted wall formula and the phenomenological reduced friction.

The dynamics of independent particles in time dependent cavities has been extensively studied earlier by Blocki and his coworkers 15 22]. Considering classical particles in vibrating cavities of various shapes, a strong correlation between chaos in classical phase space and the efficiency of energy transfer from collective to intrinsic motion was numerically observed [15]. In particular, it was demonstrated [15,16] that while the energy transfer is much smaller than the wall formula limit in a cavity undergoing quadrupole vibration, it reaches the wall formula limit for higher multipole vibrations. Similar conclusions were also reached [18 21] when the particle motion was treated quantum mechanically, though the quantal energy transfers were found to be suppressed compared to the classical ones. In the present work, we shall demonstrate that the chaos weighted wall formula can describe the energy transferred to classical particles when a cavity is strongly deformed starting from spherical shape.

The paper is organized as follows. We shall describe the fissionlike cavity motion in the next section. A procedure to obtain the irreversible energy transfer to the gas from the cavity motion will be outlined in Sec.III. Section IV will contain a comparison between this irreversible energy transfer and the chaos weighted wall formula prediction. A summary of our main results will be presented in Sec.V where we shall also draw our conclusions.

\section{A MODEL FOR FISSIONLIKE CAVITY MOTION}

We shall consider a gas of noninteracting particles in an axially symmetric cavity which is undergoing a quadrupole deformation. The initial velocity distribution for the particles will be assumed to be uniform upto a certain maximum value $v$ similar to that of a completely degenerate Fermi gas. The deformation of the surface will be proportional to the Legendre polynomials $P_{2}$ and the surface at any instant $t$ will be defined as

$$
R(\theta, t)=\frac{R_{0}}{\lambda(t)}\left\{1+\alpha(t) P_{2}(\cos \theta)\right\}
$$

where

$$
\alpha(t)=\alpha_{0}-\alpha_{2} \cos \omega t .
$$

Here $\alpha_{0}$ and $\alpha_{2}$ are parameters which define the amplitude of deformation and $\omega$ denotes its frequency while $\lambda(t)$ is a volume conserving normalization factor. According to Eq.(2.2), the rate of change of deformation will be given as $\dot{\alpha}=\alpha_{2} \omega \sin \omega t$. The cavity is thus initially at rest with a deformation $\left(\alpha_{0}-\alpha_{2}\right)$ which subsequently changes faster with time till the deformation reaches $\alpha_{0}$ at $\omega t=\frac{\pi}{2}$. If we choose $\alpha_{0}=2.0$ at which the neck radius becomes zero, the above cavity motion can serve as a model for fission dynamics during its transition from saddle to scission. By choosing a suitable value for $\alpha_{2}$, the cavity shape at the saddle point can also be defined.

We can now evaluate Eq.(1.2) for a quadrupole deformed cavity and obtain

$$
\left(\frac{\dot{E}}{E_{0}}\right)_{C W W F}=\frac{15}{4} \alpha_{2} \eta \omega \mu(\alpha(t)) I(\alpha(t)) \sin ^{2} \omega t,
$$

where

$$
I(\alpha(t))=\frac{1}{\lambda^{4}(\alpha(t))} \int_{-1}^{+1} F(z) d z
$$

and

$$
F(z)=\frac{\left[P_{2}(z)-\frac{1}{\lambda}\left(\frac{d \lambda}{d \alpha}\right)\left(1+\alpha P_{2}(z)\right)\right]^{2}\left(1+\alpha P_{2}(z)\right)^{3}}{\left[\left(1+\alpha P_{2}(z)\right)^{2}+\alpha^{2} P_{2}^{\prime 2}(z)\left(1-z^{2}\right)\right]^{1 / 2}} .
$$


In the above equations, the adiabacity index $\eta$ is defined [15] as the ratio of the maximum speed of the surface tips to the maximum particle speed $v$ and will be given by

$$
\eta=\frac{\alpha_{2} \omega R_{0}}{v},
$$

and $E_{0}$ denotes the total initial energy of the unperturbed gas. It may further be noted that for $\alpha \ll 1$, Eq.(2.3) reduces to

$$
\left(\frac{\dot{E}}{E_{0}}\right)_{C W W F}=\frac{3}{2} \alpha_{2} \eta \omega \mu(\alpha(t)) \sin ^{2} \omega t,
$$

which was used 14] earlier for small amplitude shape oscillations. In the present work however we shall use Eq.(2.3) since we shall be dealing here with large amplitude deformations. The essential steps to obtain Eq.(2.3) can be found in the Appendix. Subsequently the energy dissipated from the wall motion after a certain interval $t$ will be obtained as

$$
\left(\frac{E_{\text {diss }}(t)}{E_{0}}\right)_{C W W F}=\frac{15}{4} \alpha_{2} \eta \int_{0}^{t} \mu\left(\alpha\left(t^{\prime}\right)\right) I\left(\alpha\left(t^{\prime}\right)\right) \sin ^{2} \omega t^{\prime} d\left(\omega t^{\prime}\right) .
$$

In order to calculate the dissipated energy using Eq.(2.8), the chaoticity $\mu(\alpha(t))$ is required for all deformations $\alpha(t)$ through which the cavity evolves with time. The chaoticity for each deformation will be obtained [13] by considering particle trajectories in a static cavity with the same deformation and distinguishing between the regular and chaotic trajectories. Following the procedure outlined in Ref. [22, 14, the chaoticity $\mu$ will be determined by uniformly sampling of the trajectories which originate from the cavity surface. Fig. 1 shows the calculated values of $\mu$ which will be subsequently employed to calculate the dissipated energy according to the chaos weighted wall formula. It may also be noted that the original wall formula dissipation will be obtained by simply putting $\mu=1$ in Eq.(2.8).

\section{REVERSIBLE AND IRREVERSIBLE ENERGY TRANSFERS}

In the dynamical calculation of energy transferred to the particles in a cavity from its surface motion, the classical equation of motion of the particles is numerically solved. To this end, the initial position and the velocity vectors (lying within a Fermi sphere of radius $v$ in velocity space) of a particle are chosen at random and the trajectory of the particle is follwed in time allowing reflections whenever it encounters the oscillating walls of the cavity [15]. By considering a gas consisting of a large (typically several thousands) number of such noninteracting particles in the cavity, the relative energy transferred to the gas $E_{\text {trans }}(t) / E_{0}$ is obtained upto an interval given by $\omega t=\frac{\pi}{2}$ at which the deformation becomes maximum. Fig. 2 shows the transferred energy calculated for different values of $\eta$. The deformation parameters chosen are $\alpha_{0}=\alpha_{2}=2.0$ which correspond to an initial spherical shape and a maximum deformation of $\alpha=2.0$ at which the neck radius is zero.

A part of the transferred energy $E_{\text {trans }}(t)$ is reversible and is elastic in nature. This reversible part arises due to the symmetries present in the single particle Hamiltonian of the system and has been discussed by Blocki et al. [1] in detail. For volume conserving systems, the reversible energy depends only on the deformation of the system and is independent of the speed of the deformation. It is of interest to note here that this property is quite distinct 15 from that of a real gas. An intrinsic randomization of particle velocities is always assumed for an idea gas in a real container giving rise to the ideal gas laws according to which the energy of a gas does not change under adiabatic volume conserving deformations. The reversibility of the transferred energy in an ideal gas in a model container depends on the degree of nonintegrability of the single particle Hamiltonian and is thus fully reversible for an integrable system. This feature is illustrated in Fig. 3 where the relative transferred energy is shown over a full cycle of deformation for a cavity undergoing harmonic spheroidal deformation. The surface of the spheroidal cavity considered is defined by

$$
\frac{x^{2}}{a^{2}(t)}+\frac{y^{2}}{a^{2}(t)}+\frac{z^{2}}{c^{2}(t)}=1
$$

where

$$
c(t)=R_{0}(1+\alpha(t))
$$


and

$$
a(t)=R_{0}(1+\alpha(t))^{-\frac{1}{2}}
$$

such that it undergoes volume conserving oscillations and particles inside such a cavity constitute an integrable system. The deformation will be given as $\alpha(t)=\alpha_{0}-\alpha_{0} \cos \omega t$, where we have used $\alpha_{0}=1.0$ in our calculation. We find in Fig. 3 that the transferred energy is fully reversible and is independent of the speed of deformation.

For mixed (partially chaotic and partially regular) systems such as cavities undergoing quadrupole deformations, a part of the transferred energy is reversible while the rest is irreversible or dissipative in nature. The chaos weighted wall formula (Eq.(1.2)) describes this irreversible part of the energy transfer. We shall therefore proceed to obtain the dissipative part from the total transferred energy as follows.

Decomposing the transferred energy $E_{\text {trans }}$ into its reversible $E_{\text {rev }}$, and the dissipative $E_{\text {diss }}$, components, we write

$$
E_{\text {trans }}(\eta, \alpha)=E_{\text {rev }}(\alpha)+E_{\text {diss }}(\eta, \alpha) .
$$

The incremental energy dissipation due to an incremental adiabaticity index will therefore be given as,

$$
\Delta E_{\text {diss }}(\Delta \eta, \alpha)=\Delta E_{\text {trans }}(\Delta \eta, \alpha)
$$

where

$$
\Delta E_{d i s s}(\Delta \eta, \alpha)=E_{d i s s}(\eta+\Delta \eta, \alpha)-E_{d i s s}(\eta, \alpha)
$$

and

$$
\Delta E_{\text {trans }}(\Delta \eta, \alpha)=E_{\text {trans }}(\eta+\Delta \eta, \alpha)-E_{\text {trans }}(\eta, \alpha) .
$$

We shall obtain $\Delta E_{\text {trans }}(\Delta \eta, \alpha)$ using Eq.(3.7) by first evaluating $E_{\text {trans }}(\eta+\Delta \eta, \alpha)$ and $E_{\text {trans }}(\eta, \alpha)$ from classical dynamical calculations. According to Eq.(3.5), this quantity will ba same as $\Delta E_{\text {diss }}(\Delta \eta, \alpha)$ which shall be compared with the chaos weighted wall formula prediction in the next section.

We should remark here that one can ideally obtain the full dissipated energy $E_{\text {diss }}(\eta, \alpha)$ from Eq.(3.4) by first calculating the reversible part as

$$
E_{\text {rev }}(\alpha)=\lim _{\eta \rightarrow 0} E_{\text {trans }}(\eta, \alpha) .
$$

This scheme is difficult to implement numerically though. As the motion becomes slower (small $\eta$ ), computation time increases prohibatively and numerical instability sets in. Further, it may be recalled that an interesting observation was made in Ref. 17] where it was shown that dynamical correlation between successive collisions of a particle with the cavity wall can substantially enhance the energy transfer rate beyond the wall formula limit for very slow motions of the wall. Thus we shall not obtain the desired reversible energy transfer from calculations at very slow wall motions. We have therefore used finite values of $\eta$ (within adiabatic limit) in the above incremental method for our purpose.

\section{RESULTS}

We shall first consider a cavity which starts deforming from a spherical shape and reaches a maximum deformation of $\alpha=2.0$. The relative energy transferred to the gas for this system at different values of $\eta$ is given in Fig. 2 . Following the procedure outlined in the preceeding section, the incremental energy dissipation for various values of $\Delta \eta$ are then obtained. This energy dissipation is also calculated from both the chaos weighted wall formula and the original wall formula using Eq. 2.8). Fig. 团 shows the comparison. It is observed that the chaos weighted wall formula prediction for energy dissipation is very close to that from dynamical calculation. On the other hand, the original wall formula overestimates the energy dissipation by more than $50 \%$.

We shall next consider a cavity for which we set $\alpha_{0}=2.0$ and $\alpha_{2}=1.5$. This gives rise to an initial deformation of $\alpha=0.5$ at $t=0$. The results of dynamical calculations for energy transfer are shown in Fig. 5 . The incremental energy dissipations are subsequently obtained and the comparison with predictions of the chaos weighted wall formula and the original wall formula is shown in Fig. 6. The chaos weighted wall formula prediction remains reasonably close to the dynamically calculated values for lower values of $\Delta \eta(0.02$ and 0.04$)$. For higher values of $\Delta \eta(0.06$ and 0.08), the agreement becomes somewhat poorer at larger deformations. Nevertheless, the chaos weighted wall formula 
provides a much better description of the dynamical results compared to the original wall formula for all the cases considered here.

We shall now calculate the reduced friction coefficient which corresponds to the dissipation according to the chaos weighted wall formula. The reduced friction coefficient $\beta$ is defined [f] as $\beta=\gamma / M$ where

$$
\dot{E}=\gamma \dot{q}^{2} R_{0}^{2} .
$$

Here $q$ is half the distance between the centres of mass of the equal fragments on each side of the neck divided by $R_{0}, M$ is the total mass of the gas and $\gamma$ is the friction coefficient. The value of $q$ varies from $q=0.375$ for spherical configuration to $q=1.0$ for scission. The friction coefficient $\gamma$ and hence the reduced friction coefficient $\beta$ can be evaluated from Eq.(2.8) for both the chaos weighted wall formula and the original wall formula $(\mu=1)$. The reduced friction coefficients thus obtained are plotted in Fig. 8 . The phenomenological reduced friction coefficient introduced by Fröbrich [6,7] is also shown in this figure.

We note in Fig. 7 that the friction strength is strongly suppressed in chaos weighted wall formula for spherical or near spherical configurations. This reduction in the dissipative force at the early stages of nuclear fission is an important consequence of the chaos weighted wall formula. It is of interest to note that a reduction of the wall plus window formula of one body dissipation was also suggested [3 5] earlier. Further, such a reduction was phenomenologically found [7] to be essential in order to reproduce the excitation functions of both the prescission neutron multiplicities and the fission probabilities. The subsequent increase of the reduced friction coefficient (due to chaos weighted wall formula) at larger values of $q$ essentially reflects the increase of chaoticity with deformation. Since chaoticity depends sensitively on the nonintegrability of the system, the reduced friction coefficient at large $q$ in turn depends on the details of the shape considered. Thus the comparison of our present calculation for a model shape evolution with the phenomenological friction is a qualitative one, both showing an increase of the reduced friction at higher deformations starting with very small values at the spherical configuration. We further note that the phenomenological friction near scission is much stronger than the original wall or its present chaos weighted version (both merge near scission). This can possibly be understood [8] if other effects such as the exchange mechanism ("window" formula) and temperature dependence of nuclear friction are considered.

\section{SUMMARY AND CONCLUSIONS}

In the preceding sections, we have applied the chaos weighted wall formula to an ideal gas in a cavity undergoing fissionlike shape evolution in order to test the validity of the former for large amplitude motions. To this end, we have dynamically calculated the energy transferred from the wall to the gas and compared its irreversible part with the prediction of the chaos weighted wall formula. In order to simulate the macroscopic dynamics of symmetric fission in our model, we have chosen a cavity initially at rest with an initial deformation which is then progressivly deformed till the neck radius vanishes. In all the cases considered above, we find a fair agreement between the results from dynamical calculations and the predictions of the chaos weighted wall formula. The original wall formula is found to considerably overestimate the energy transfer.

We have also compared the reduced friction coefficient extracted from the chaos weighted wall formula with that prescribed [7] phenomenologically in order to reproduce the excitation functions of both the precission neutron multiplicities and the fission probabilities. Both the friction coefficients are found to be qualitatively similar. An important feature of the phenomenological friction is its strong suppression for compact shapes which is also present in the chaos weighted wall formula.

We can therefore conclude that the chaos weighted wall formula provides a fairly accurate description of one body dissipation in dynamical systems similar to fissioning nuclei. Further, the qualitative similarity between the phenomenological friction in nuclear fission and the chaos weighted wall formula provides evidence for one body nature of the dissipative force acting in a fissioning nucleus.

\section{ACKNOWLEDGEMENTS}

The authors are grateful to Professor Jan Blocki for sending us the computer codes for classical trajectory calculations which have been used extensively in this work. 


\section{APPENDIX A:}

We shall briefly outline here the essential steps to arrive at the wall formula dissipation rate for cavities with large quadrupole deformations which has been used in Eq.(2.3).

The wall formula is given as,

$$
\dot{E}=\rho \bar{v} \int \dot{n}^{2} d \sigma,
$$

where $\dot{n}=$ normal component of the velocity of the surface element $d \sigma$ at a point $\vec{r}=r(\theta, \phi)$. The wall of an azimuthally symmetric quadrupole cavity is defined by,

$$
r(\theta, t)=\frac{R_{0}}{\lambda(t)}\left[1+\alpha(t) P_{2}(\cos \theta)\right] .
$$

Taking the surface velocity component along the normal,

$$
\dot{n}=\dot{r} \cos \theta_{n},
$$

where $\theta_{n}$ is the angle between $\vec{r}$ and $\vec{n}$ and is given by,

$$
\cos \theta_{n}=r \frac{d \theta}{d s}=\frac{r}{\left[r^{2}+\left(\frac{d r}{d \theta}\right)^{2}\right]^{1 / 2}},
$$

and where we have used,

$$
d s=d \theta\left[r^{2}+\left(\frac{d r}{d \theta}\right)^{2}\right]^{1 / 2} .
$$

Hence

$$
\dot{E}=2 \pi \rho \bar{v} \int \frac{\dot{r}^{2} r^{3}}{\left[r^{2}+\left(\frac{d r}{d \theta}\right)^{2}\right]^{1 / 2}} \sin \theta d \theta
$$

Now from Eq. (A2) we get,

$$
\dot{r}=\frac{R_{0}}{\lambda}\left[\dot{\alpha} P_{2}-\frac{\dot{\lambda}}{\lambda}\left(1+\alpha P_{2}\right)\right],
$$

and

$$
\frac{d r}{d \theta}=-\frac{R_{0}}{\lambda} \alpha P_{2}^{\prime}(z) \sin \theta,
$$

where $P_{2}^{\prime}(z)=d P_{2}(z) / d z$ and $z=\cos \theta$. Using the above in Eq.(A4), we obtain

$$
\dot{E}=\frac{2 \pi \rho \bar{v} R_{0}^{4} \dot{\alpha}^{2}}{\lambda^{4}} \int_{-1}^{+1} F(z) d z
$$

where

$$
F(z)=\frac{\left[P_{2}(z)-\frac{1}{\lambda}\left(\frac{d \lambda}{d \alpha}\right)\left(1+\alpha P_{2}(z)\right)\right]^{2}\left(1+\alpha P_{2}(z)\right)^{3}}{\left[\left(1+\alpha P_{2}(z)\right)^{2}+\alpha^{2} P_{2}^{\prime 2}(z)\left(1-z^{2}\right)\right]^{1 / 2}} .
$$

The above equations give the rate of energy transfer for large quadrupole deformations. 
[1] J.Blocki, Y.Boneh, J.R.Nix, J.Randrup, M.Robel, A.J.Sierk and W.J.Swiatecki, Ann.Phys.(N.Y.)113, 330(1978).

[2] A.J. Sierk and J.R. Nix, Phys. Rev. C21, 982(1980).

[3] J.R. Nix and A.J. Sierk, Proc. International School-Seminar on Heavy Ion Physics, Dubna, USSR, 1986, Report No. JINR-D7-87-68(1987), p.453.

[4] J.R. Nix and A.J. Sierk, Proc. International Symposium on Perspectives in Nuclear Physics, Madras, India, 1987, J. Madras University B50,38(1987).

[5] J.R. Nix and A.J. Sierk, Proc. 6th Adriatic Conference on Nuclear Physics: Frontiers of Heavy Ion Physics, Dubrovnik, Yogoslavia, 1987, eds. N. Cindro et al. (World Scientific, Singapore, 1990) p.333.

[6] P.Fröbrich, I.I. Gontchar, Nucl.Phys.A563, 326(1993).

[7] P.Fröbrich, I.I. Gontchar and N.D. Mavlitov, Nucl.Phys.A556, 281(1993).

[8] Y. Abe, S.Ayik, P. -G. Reinhard and E. Suraud, Phys.Rep. 275,49(1996).

[9] J. Blocki and J. Wilczyński, Acta Phys. Pol. B28, 133(1997).

[10] S.E.Koonin and J.Randrup, Nucl.Phys.A289, 475(1977).

[11] C. Yannouleas, Nucl.Phys.A439, 336(1985).

[12] J.J. Griffin and M. Dworzecka, Nucl.Phys.A455, 61(1986).

[13] Santanu Pal and Tapan Mukhopadhyay, Phys.Rev. C54, 1333(1996).

[14] Tapan Mukhopadhyay and Santanu Pal, Phys.Rev. C56, 296(1997).

[15] J.Blocki, J.-J.Shi and W.J.Swiatecki, Nucl.Phys. A554, 387(1993).

[16] J. Blocki, F. Brut and W.J. Swiatecki, Nucl.Phys.A554, 107(1993).

[17] J. Blocki, C. Jarzynski and W.J. Swiatecki, Nucl.Phys.A599, 486(1996).

[18] J. Blocki, F. Brut and W.J. Swiatecki, Acta Phys. Pol.B25, 637(1994).

[19] J. Blocki, J. Skalski and W.J. Swiatecki, Nucl.Phys.A594, 137(1995).

[20] J. Blocki, J. Skalski and W.J. Swiatecki, Nucl.Phys.A618, 1(1997).

[21] P. Magierski, J. Skalski and J. Blocki, Phys.Rev.C56, 1011(1997).

[22] J. Blocki, F. Brut, T. Srokowski and W.J. Swiatecki, Nucl.Phys.A545, 511c(1992).

FIG. 1. Variation of the chaoticity with deformation for quadrupole shapes. Solid circles are calculated values and the line is to guide the eye.

FIG. 2. Growth of relative energy transferred to the gas from the wall motion. Curves $a, b, c, d$ and $e$ in the lower panel are for $\eta=0.02,0.04,0.06,0.08$ and 0.10 respectively while curves $f, g, h$ and $i$ in the upper panel are for $\eta=0.03,0.05,0.07$ and 0.09 respectively. The cavity motion is defined by $\alpha_{0}=\alpha_{2}=2.0$.

FIG. 3. Relative energy growth for a cavity undergoing spheroidal deformation showing the complete reversibility of the energy transferred. The plot represents calculated values for $\eta=0.02,0.04,0.06,0.08$ and 0.10 which are all indistinguishable from each other.

FIG. 4. Growth of incremental dissipated energy relative to $E_{0}$ for different values of $\Delta \eta$. The solid lines are from dynamical calculation. The long dashed and short dashed lines are the original wall formula (WF) and chaos weighted wall formula (CWWF) predictions respectively.

FIG. 5. Same as Fig.2 but for the cavity motion defined by $\alpha_{0}=2.0$ and $\alpha_{2}=1.5$.

FIG. 6. Same as Fig.4 but for the cavity motion defined by $\alpha_{0}=2.0$ and $\alpha_{2}=1.5$.

FIG. 7. Reduced friction coefficients from chaos weighted wall formula (solid line), original wall formula (long dashed line) and from Ref.[7] (short dashed line). 


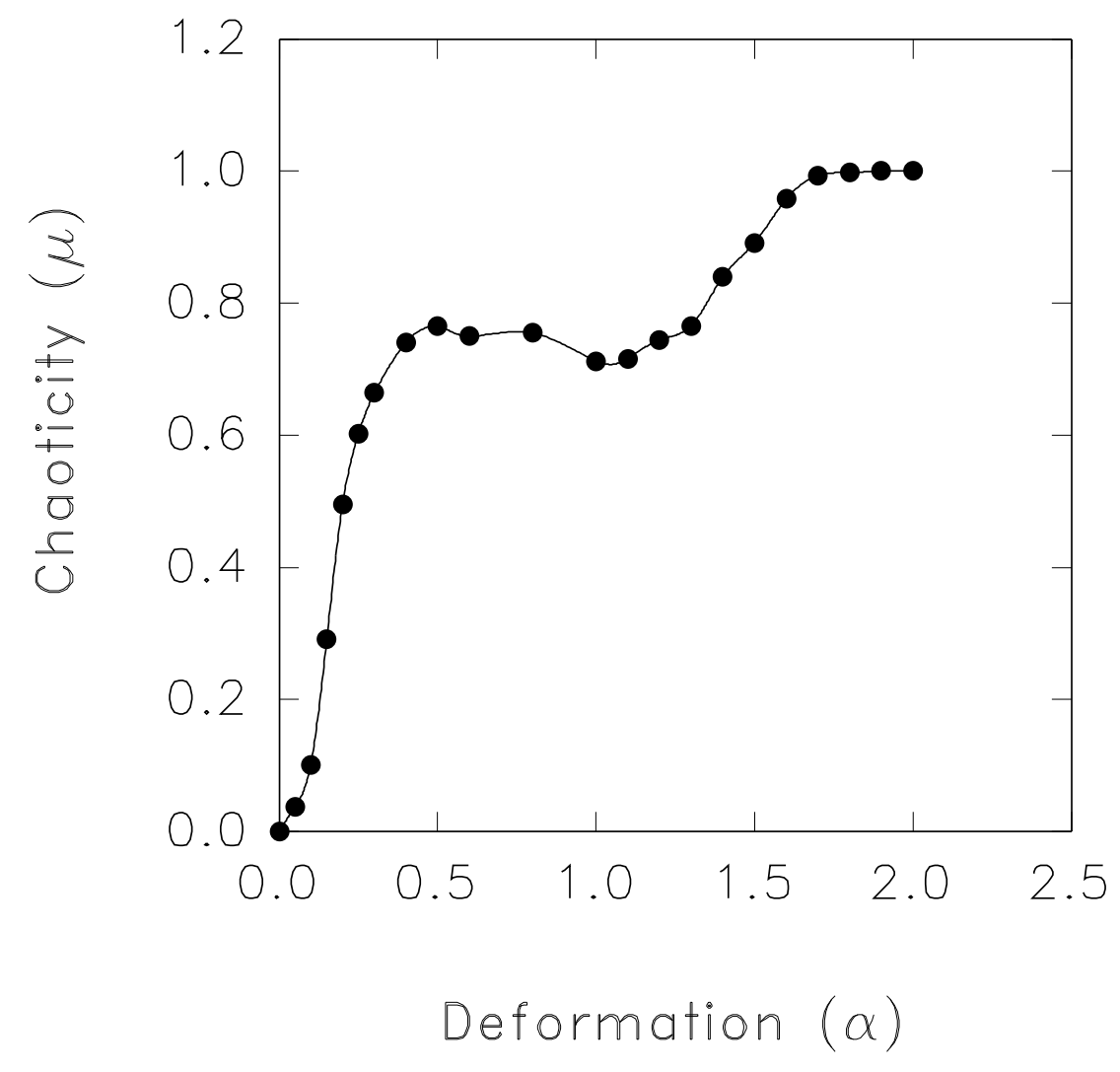

Fig. 1 


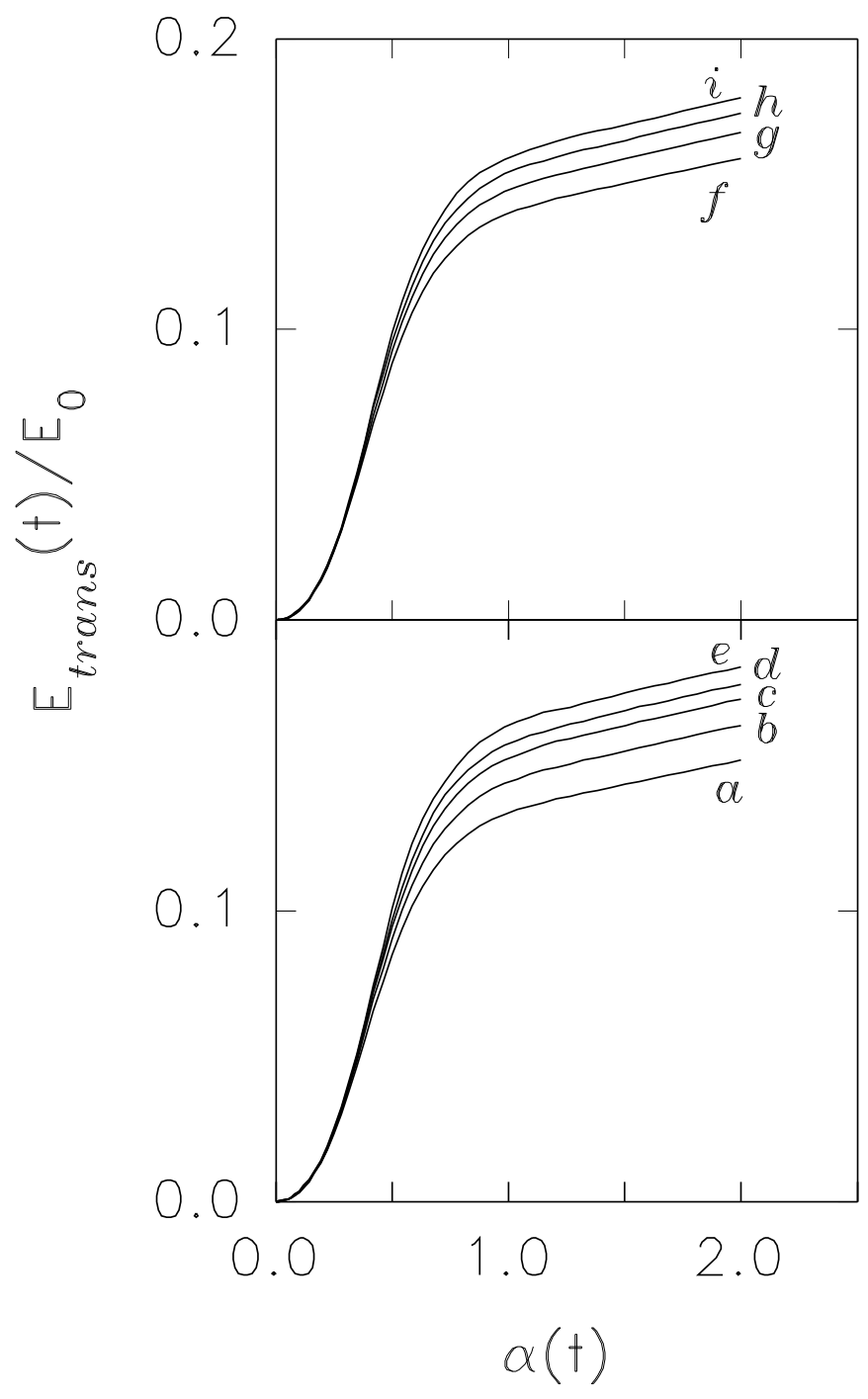

Fig. 2 


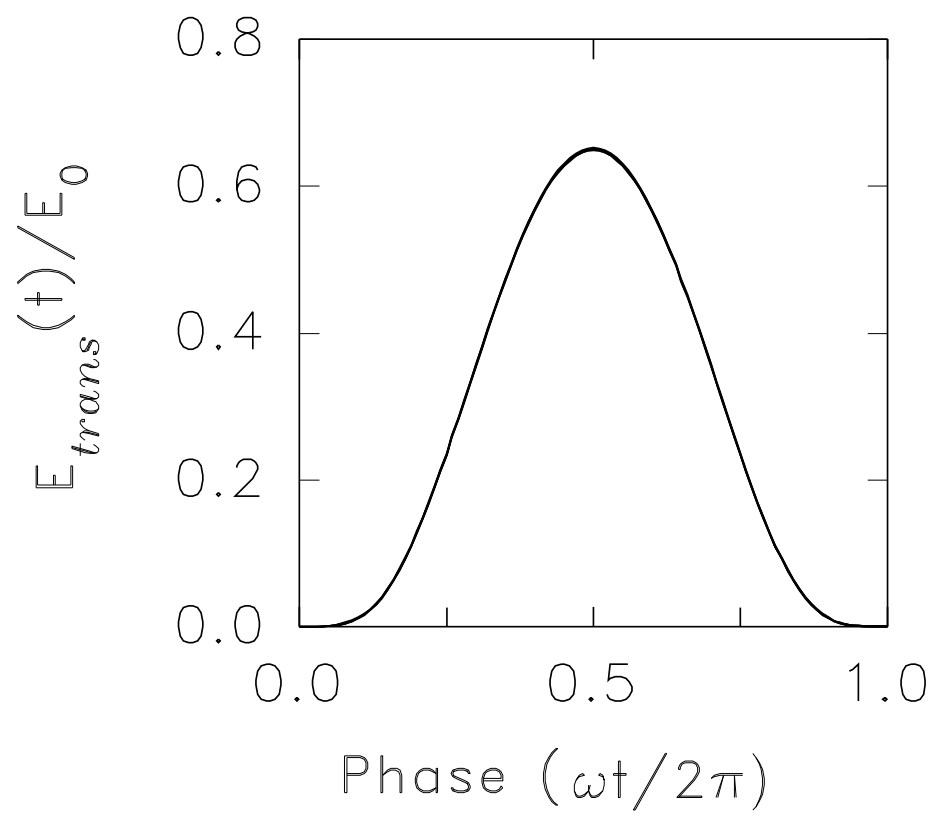

Fig. 3 


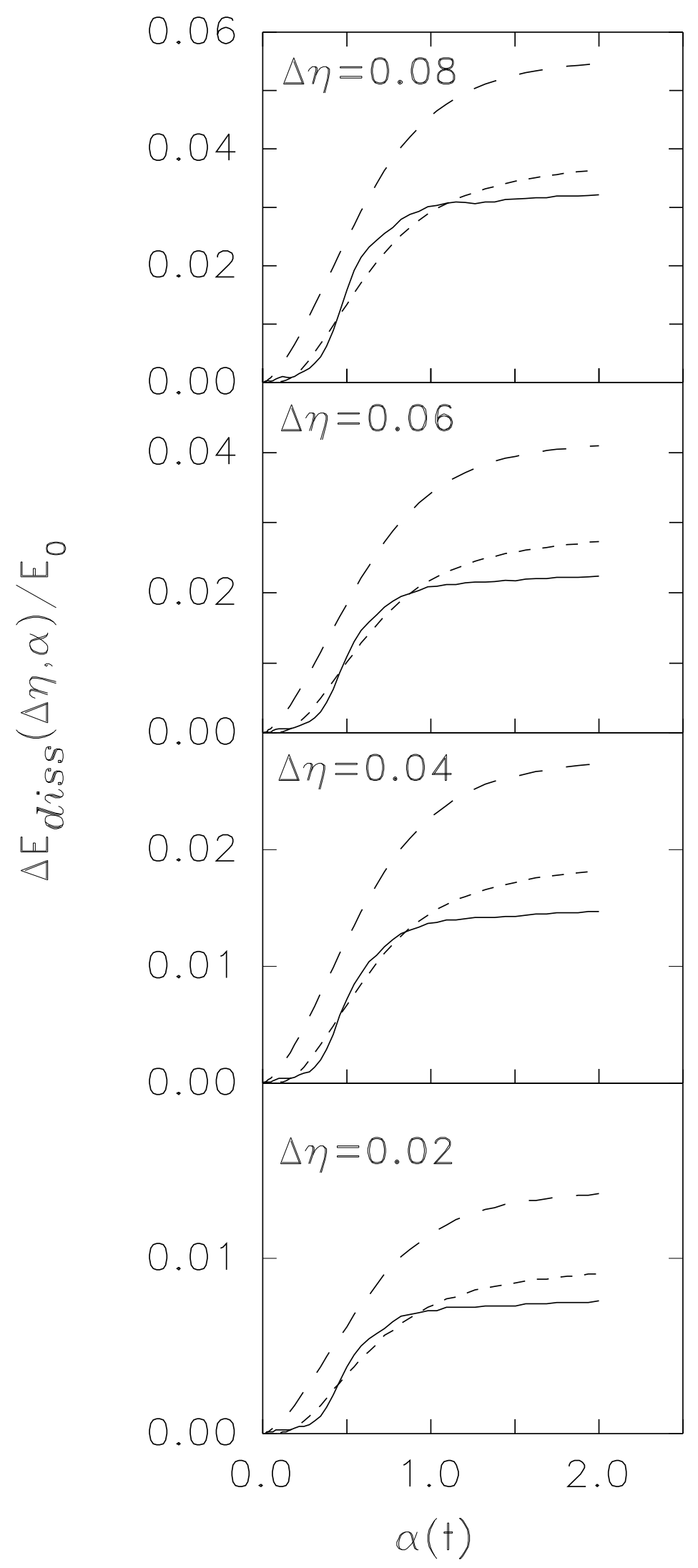

Fig. 4 


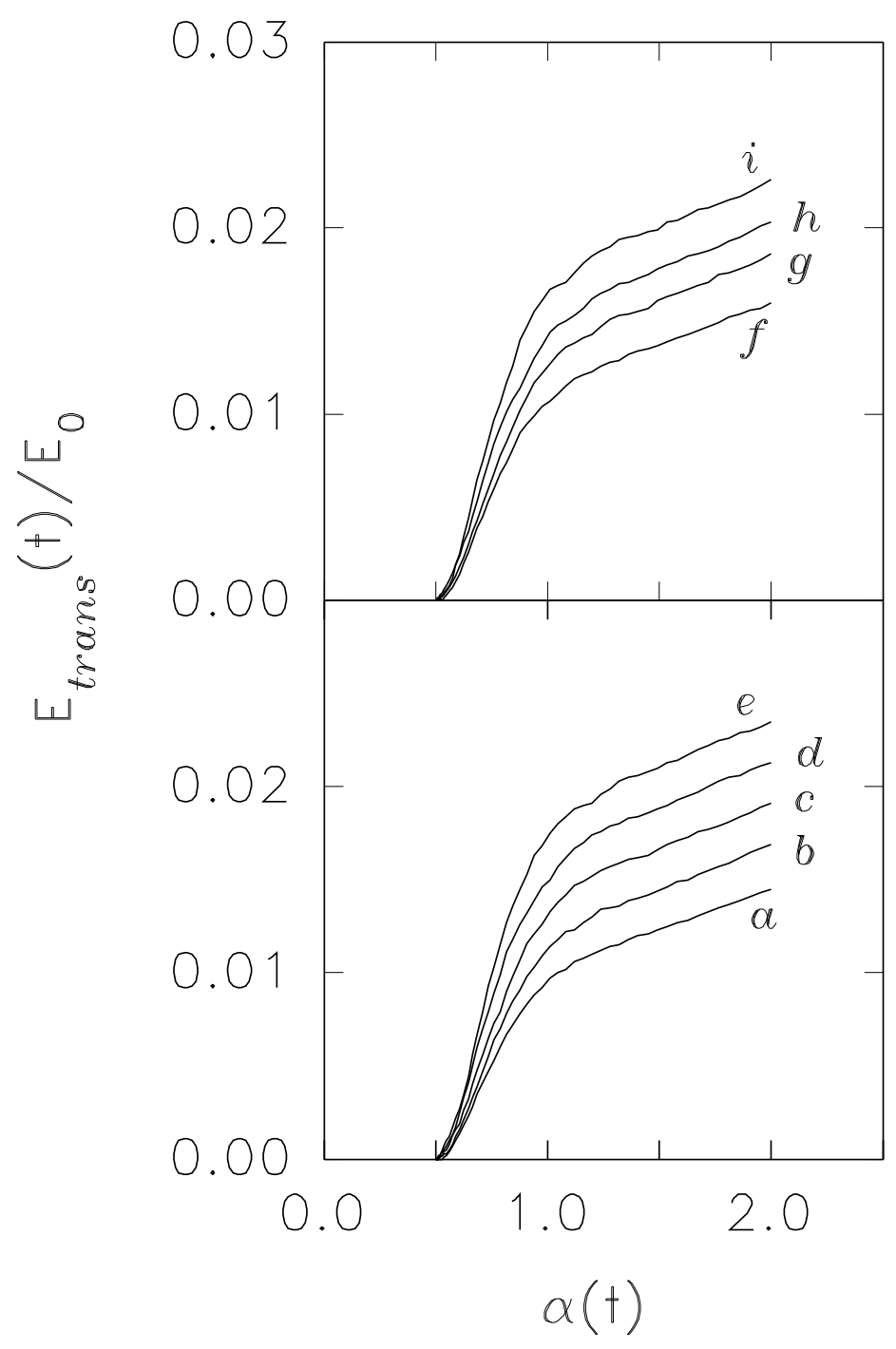

Fig. 5 


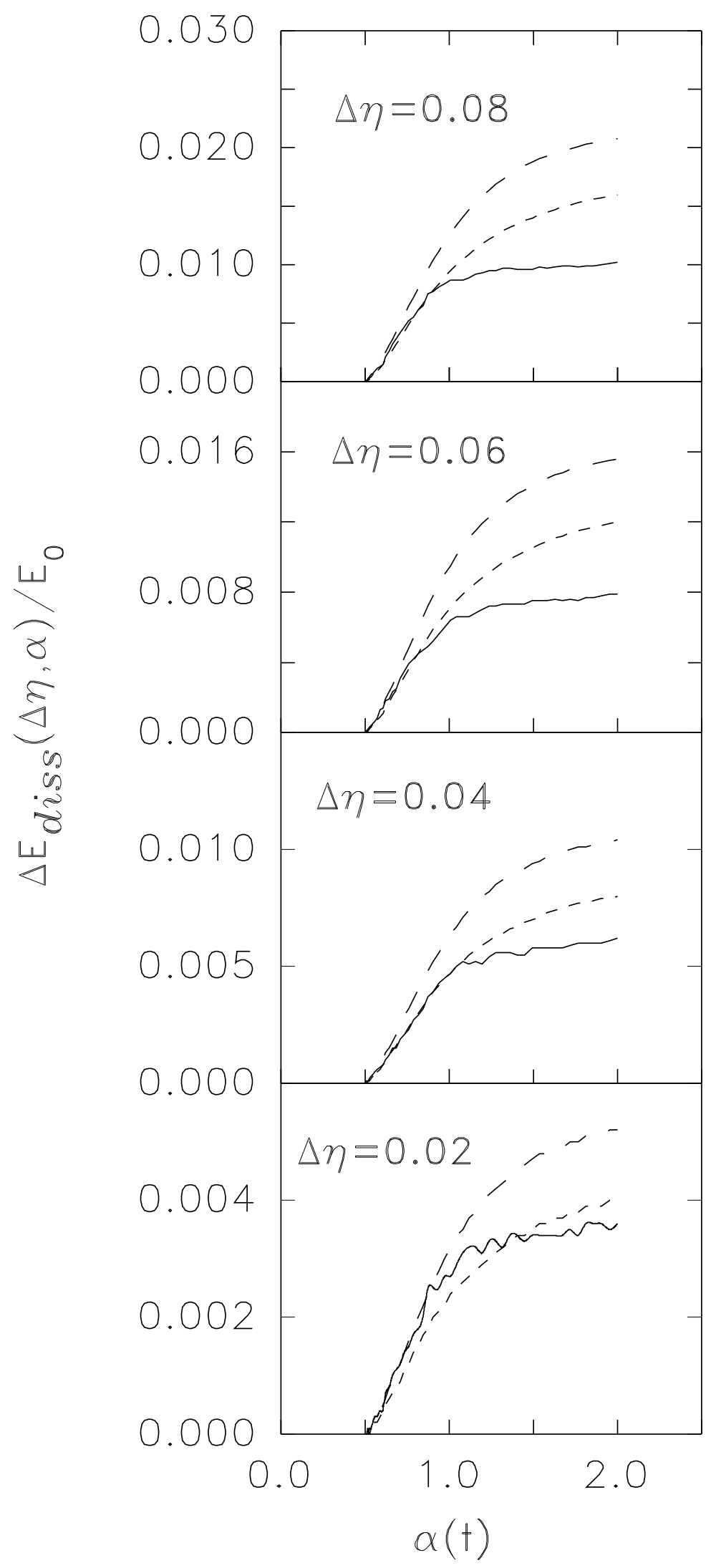

Fig. 6 


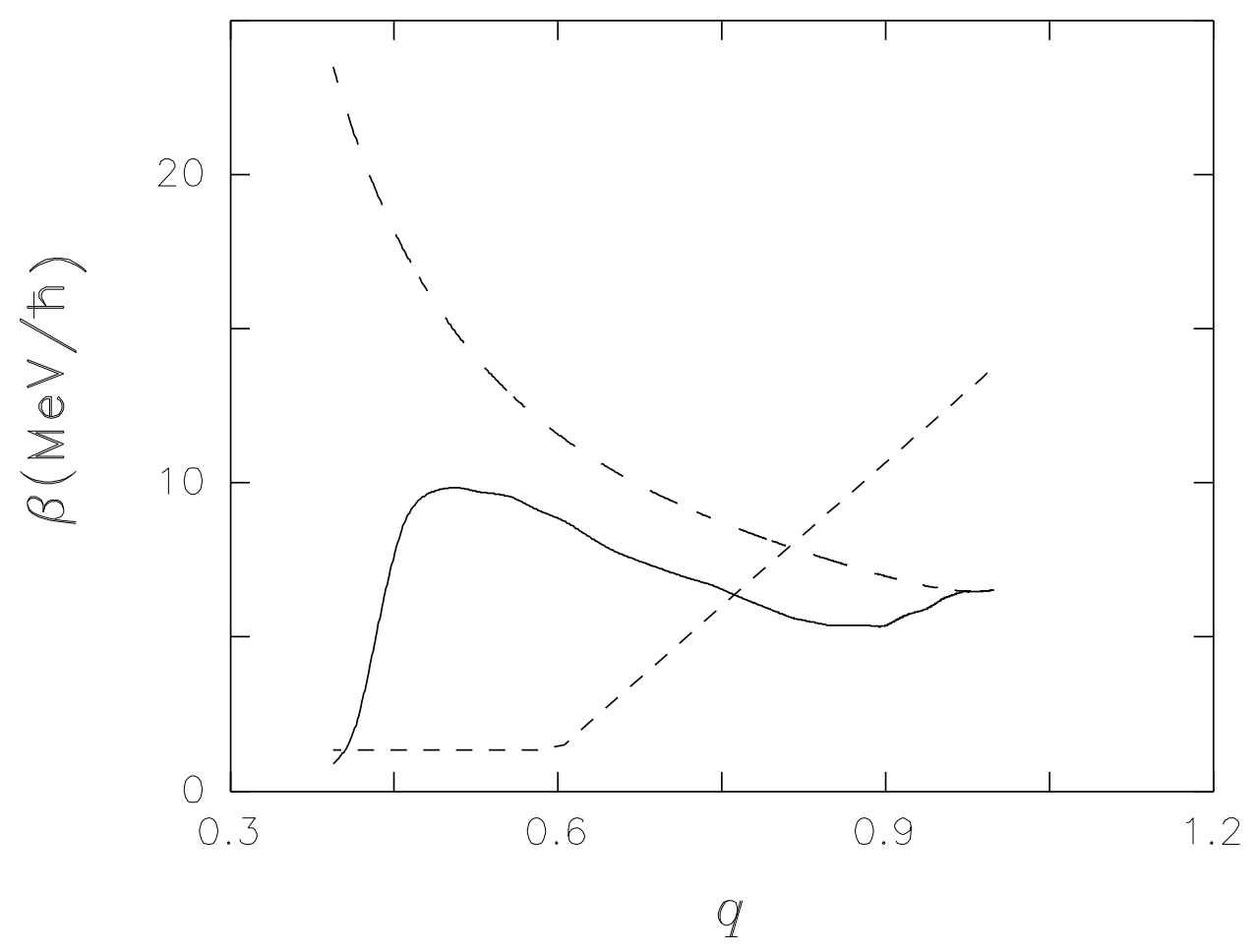

Fig. 7 